\title{
Mental health services: quality, safety and suicide
}

\author{
Nav Kapur (D) ,1,2,3 Louise S Gorman, ${ }^{1,2}$ Leah Quinlivan, ${ }^{1,2}$ \\ Roger T Webb ${ }^{1,2}$
}

${ }^{1}$ NIHR Greater Manchester Patient Safety Translational Research Centre, The University of Manchester, Manchester Academic Health Science Centre, Manchester, UK, Manchester, UK ${ }^{2}$ Centre for Mental Health and Safety, University of Manchester, Manchester, UK, The University of Manchester, Manchester, UK ${ }^{3}$ Greater Manchester Mental Health NHS Foundation Trust, Manchester, UK

\section{Correspondence to} Professor Nav Kapur, Centre for Mental Health and Safety, University of Manchester, Manchester, UK, Manchester, Greater Manchester M13 9PL, UK:

nav.kapur@manchester.ac.uk

Accepted 25 September 2021

\section{Sinked}

- http://dx.doi.org/10.1136/ bmjqs-2020-012944

- http://dx.doi.org/10.1136/ bmjqs-2020-012975

\section{Check for updates}

(c) Author(s) (or their employer(s)) 2021. No commercial re-use. See rights and permissions. Published by BMJ.

To cite: Kapur N, Gorman LS, Quinlivan L, et al.

BMJ Qual Saf Epub ahead of print: [please include Day

Month Year]. doi:10.1136/

bmjqs-2021-013532
Suicide is a major global challenge with an estimated 700000 people taking their lives each year. ${ }^{1}$ Each of these deaths is an individual tragedy affecting families, friends, communities and health and social care teams. As clinicians and researchers working in suicide prevention, we are sometimes contacted by people whose loved ones have died by suicide while under the care of mental health services. Although we hear about examples of high-quality care, there are also accounts of poor continuity, failed communication, diagnostic or therapeutic errors, poorly trained or resourced clinical teams or a lack of family involvement. Suicide is a complex phenomenon and many of its drivers are economic and social, ${ }^{2}$ but its prevention should be a priority for health services in general and for mental health services in particular. Specifically, mental health patients represent a group at greatly elevated risk of suicide who are accessible because they are in contact with services. $^{3}$

Two studies, carried out in the Veterans Health Administration (VHA) in the USA and published in this issue of BMJ Quality and Safety, have explored the relationship between different aspects of mental health provision and suicide risk. The paper by Kaboli and colleagues ${ }^{4}$ investigated the association between mental health bed occupancy and the veterans' suicide rate in the catchment areas of 111 VHA hospitals across 50 states over a 5 -year period. The study found that areas with inpatient psychiatric units operating at the greatest occupancy levels had higher suicide rates than areas with the lowest occupancy levels. As the authors point out, these results make clinical sense. It is plausible that 'hospital strain' and equivalent pressures in community care make some services less safe than others. It may well be that occupancy levels are an important safety metric. Although we did not find an association between occupancy and suicide in a previous study, ${ }^{5}$ it remains a live issue in the UK with the Royal College of Psychiatrists recommending a maximum mental health bed occupancy of $85 \%$. However, we should treat the findings of the Kaboli et al paper with some caution. This was an ecological study and an alternative explanation is that that high levels of bed occupancy in certain areas simply reflected higher underlying clinical and social need, and by implication, higher suicide risk. The researchers do make the point that any unmeasured confounding influences would need to be large to wholly account for the observed associations.

By contrast, in their study of all 115 VHA mental health referral regions, Shiner and colleagues ${ }^{6}$ found no relationship between the quality of VHA mental health outpatient care (using a composite quality measure that they developed) and area-level suicide rates. What should we make of this? That outpatient mental health services have no role in the prevention of suicide? Again, we should be cautious in how we interpret these findings. Crude quality metrics based on selected aggregated data may not reflect how real-world care is delivered to individual patients, and these metrics may represent only some aspects of care that may reduce the risk for suicide. Like the previously cited paper, ${ }^{4}$ this study also used an ecological design that could not examine the influence of individual-level confounders, although the researchers did attempt to control for underlying area-based risks by adjusting for population suicide rates. ${ }^{6}$ Perhaps most importantly, the investigators were seeking to observe a relationship between mental health outpatient provision and suicide rates in the wider VHA population. This 
may have been an unrealistic aim. Our own data from the UK suggests that only $25 \%-30 \%$ of people who die by suicide are under the care of specialist mental health services. ${ }^{3}$ Thus, the study may have better placed to find any existing association if they had looked directly at the relationship between the quality of mental health outpatient services and the suicide rate in people in receipt of those services.

In the UK, we have been collecting detailed clinical data on every mental health patient who has died by suicide over the past 25 years as part of the National Confidential Inquiry into Suicide and Safety in Mental Health (NCISH). What have we learnt? The sheer number of such deaths is alarming-over 37000 individuals have died by suicide while under the care of mental health services since the Inquiry began, with around half of these patients having mental health contact in the week before they died. ${ }^{3}$

NCISH research has shown that focussing on safety in specific mental health settings can be beneficial. In the UK, as in many other countries, the psychiatric bed-base has shrunk considerably over time. This means that the people who are admitted tend to be more unwell and have more complex needs than they did in the past. These temporal factors might be expected to have increased the inpatient suicide rate, yet it has in fact fallen to the lowest levels on record, probably as a result of a focus on environmental safety (eg, the removal of potential ligature points) and greater vigilance when inpatients leave the ward. ${ }^{3}$ However, mental health services need to take care that the risk is not simply transferred to other settings. There was some evidence for an increase in suicide between 1997 and 2008 in the immediate postdischarge period or in those being managed under Crisis Resolution Home Treatment Teams (as an alternative to inpatient admission). ${ }^{7}$ A safety focus needs to apply across the care pathway. As recommended by the authors of a recent paper based on VHA postsuicide incident reports, different hospital and community settings need specific suicide prevention measures. ${ }^{8}$

System-wide changes in mental health services can also improve safety. In a national before-and-after study conducted using NCISH data, we found that changes to mental health service provision designed to improve safety were indeed associated with lower suicide rates. ${ }^{9}$ For three of the service changes in particular, there were large reductions in suicide risk. These were: implementation of 24-hour crisis care; having a dual diagnosis policy (that is a specific policy for treating patients with mental illness in the context of drug or alcohol misuse, perhaps a marker of clinical activity in this area) and having a system of multidisciplinary review after suicide (perhaps an indicator of a learning culture). The reduction in suicide numbers was clinically important with around 200-300 fewer suicide deaths per year. ${ }^{9}$ However, these reductions were not observed in services that did not implement the changes.

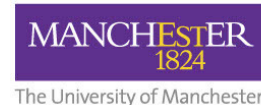

$\mathrm{NCISH}:$

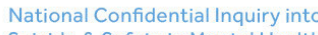
Suicide \& Safety in Mental Health

The University of Manchester

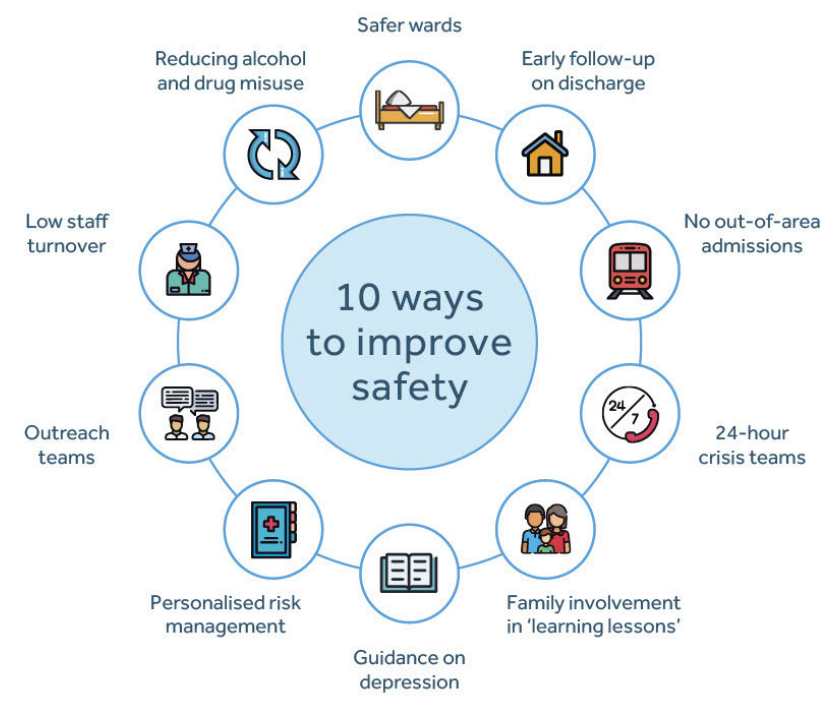

www.manchester.ac.uk/ncish

Figure 110 ways to improve mental health safety (from https://sites. manchester.ac.uk/ncish/).

Organisational factors are important too. In a later $\mathrm{NCISH}$ study, we found that suicide occurred more frequently in those mental health services with a greater nursing staff turnover. ${ }^{5}$ Intriguingly, we found that the organisational factors appeared to interact with individual service changes. Specifically, implementing service changes in services that were organisationally healthy seemed to have a greater suicide prevention impact than implementing them in services with organisational problems. ${ }^{5}$ To optimally improve mental health patient safety, we therefore need to attend to both individual service factors and the organisational context in which care is provided. Safety, like suicide, is complex and the factors that influence risk go wider than those evaluated in the two featured studies. ${ }^{4} 6$ Figure 1 summarises the key preventive approaches that have been informed by NCISH research.

Of course, any safety focus needs to extend beyond specialist mental health services. As many as $40 \%$ of people who die by suicide will have visited a hospital emergency department during the preceding 12 months ${ }^{10}$ and over $60 \%$ will have been seen in primary care. ${ }^{11}$ We also need to understand that suicide is not the only marker of mental health safety. Non-fatal self-harm, which includes intentional self-poisoning or injury regardless of the degree of suicidal intent, is the strongest risk factor for suicide and many of the strategies for suicide intervention and prevention are common to both. ${ }^{12}$ Doing simple things well, such as providing high-quality assessments to people when they present to services after a self-harm episode, ${ }^{13}$ or 
making psychological treatments readily available, ${ }^{14}$ reduces the risk of self-harm repetition and is also likely to help prevent suicide.

Access to services is important but so is having services that are fit for purpose. Middle-aged men have a greatly elevated suicide rate in the UK and in other countries. The accepted wisdom is that one of the reasons for this is that men of this age do not seek help and 'don't talk'. In fact, in a recent national study, we found that $90 \%$ of 242 middle-aged men who died by suicide had been in contact with health or social care services. ${ }^{15}$ Interestingly, although $44 \%$ were prescribed antidepressants, only 5\% were receiving psychological intervention. As well as encouraging men to seek help, services need to ensure that they meet men's needs when they do come forward.

There are major gaps in our understanding of mental health patient safety. Mental health services have been transformed by the COVID-19 pandemic in many countries, with evidence of reduced activity, greater acuity and moves towards unevaluated remote ways of delivering healthcare during the acute phase. ${ }^{16}$ The long-term impact of these phenomena is uncertain. We also know next to nothing about mental health patient safety in low-income and middle-income countries. Safety challenges will not be the same across patient groups or settings. In particular, there are concerns about suicide and self-harm in young people, ${ }^{17}$ in people from ethnic minority groups, ${ }^{18}$ and in those who are socioeconomically deprived. ${ }^{19}$ Safety in psychological therapy services has been relatively under-researched. This has come into sharper focus recently with the expansion of such services in some countries-for example under the Increasing Access to Psychological Therapies (IAPT) programme in England. The role of innovative, technology-assisted assessment (eg, machine learning, artificial intelligence) should be explored, although risk prediction for suicide is an unrealistic goal given the poor predictive ability of even the best assessment tools and relatively low base rate of target behaviours even within mental health populations. ${ }^{20}$

Where should our mental health safety focus be in the years to come? Perhaps for once, this is not just about 'needing further research' but better implementation of what we know. As part of the National Institute for Health Research Greater Manchester Patient Safety Translational Research Centre, we are exploring barriers to more effective involvement of family members in mental health crisis service provision and barriers to provision of evidence-based care for people who have harmed themselves. ${ }^{21}$ As part of major NHS England programmes, we are helping local areas to use and implement the evidence-base for suicide and self-harm prevention. ${ }^{22}$ Focussing on areas of positive practice and fostering an optimal learning culture within services ${ }^{23}{ }^{24}$ may also help to ensure that we provide the safest possible care to every patient who seeks help for their mental health problems.

Acknowledgements We thank Donna Littlewood for her contribution to the mental health projects described in this paper. We would also like to thank our patient and public advisory panel (MS4MH-R) for their involvement throughout the research programme.

Contributors NK wrote the editorial with input from LSG, LQ and RTW. All authors approved the final version.

Funding This work was supported by the National Institute for Health Research (NIHR) Greater Manchester Patient Safety Translational Research Centre (reference number: PSTRC-2016-003). The National Confidential Inquiry into Suicide and Safety in Mental Health (NCISH) is funded by the Healthcare Quality Improvement Partnership (HQIP). NK is also supported by the University of Manchester, Greater Manchester Mental Health NHS Foundation Trust. All researchers are independent from the funders.

Disclaimer The funders had no role in study design, data collection and analysis, interpretation, decision to publish, or preparation of the manuscript. The views expressed are those of the authors' and not necessarily those of the NIHR or the Department of Health and Social Care, HQIP, NHS England, or NICE.

Competing interests NK chaired the NICE guidelines for selfharm (longer term management) 2012 and the NICE Quality Standards on self-harm 2013. He is currently topic advisor for the new NICE guidelines for self-harm and chairs the NICE depression guideline committee. He works with NHS England on national quality improvement initiatives for suicide and self-harm and sits on the Department of Health and Social Care's National Suicide Prevention Strategy Advisory Group for England.

Patient consent for publication Not required.

Provenance and peer review Commissioned; internally peer reviewed.

ORCID ID

Nav Kapur http://orcid.org/0000-0002-3100-3234

\section{REFERENCES}

1 World Health Organization. Suicide worldwide in 2019: global health estimates. Geneva: World Health Organization, 2021Licence: CC BY-NC-SA 3.0 IGO. https://www.who.int/ teams/mental-health-and-substance-use/suicide-data

2 Chang S-S, Stuckler D, Yip P, et al. Impact of 2008 global economic crisis on suicide: time trend study in 54 countries. BMJ 2013;347:f5239.

3 National Confidential Inquiry into Suicide and Safety in Mental Health. Annual report: England, Northern Ireland, Scotland and Wales. Manchester: The University of Manchester, 2021.

4 Kaboli PJ, Augustine MR, Haraldsson B, et al. Association between acute psychiatric bed availability in the Veterans health administration and veteran suicide risk: a retrospective cohort study. BMJ Qual Saf 2021. doi:10.1136/ bmjqs-2020-012975. [Epub ahead of print: 16 Aug 2021].

5 Kapur N, Ibrahim S, While D, et al. Mental health service changes, organisational factors, and patient suicide in England in 1997-2012: a before-and-after study. Lancet Psychiatry 2016;3:526-34.

6 Shiner B, Gottlieb DJ, Levis M, et al. National cross-sectional cohort study of the relationship between quality of mental healthcare and death by suicide. BMJ Qual Saf 2021. 
7 Kapur N, Hunt IM, Windfuhr K, et al. Psychiatric in-patient care and suicide in England, 1997 to 2008: a longitudinal study. Psychol Med 2013;43:61-71.

8 Mills PD, Soncrant C, Gunnar W. Retrospective analysis of reported suicide deaths and attempts on veterans health administration campuses and inpatient units. BMJ Qual Saf 2021;30:567-76.

9 While D, Bickley H, Roscoe A, et al. Implementation of mental health service recommendations in England and Wales and suicide rates, 1997-2006: a cross-sectional and before-andafter observational study. Lancet 2012;379:1005-12.

10 Gairin I, House A, Owens D. Attendance at the accident and emergency department in the year before suicide: retrospective study. Br J Psychiatry 2003;183:28-33.

11 Windfuhr K, While D, Kapur N, et al. Suicide risk linked with clinical consultation frequency, psychiatric diagnoses and psychotropic medication prescribing in a national study of primary-care patients. Psychol Med 2016;46:3407-17.

12 Kapur N. Services for self-harm: progress and promise? Br J Psychiatry 2020;217:663-4.

13 Kapur N, Steeg S, Webb R, et al. Does clinical management improve outcomes following self-harm? results from the multicentre study of self-harm in England. PLoS One 2013;8:e70434.

14 Hawton K, Witt KG, Taylor Salisbury TL. Psychosocial interventions for self-harm in adults. Cochrane Database Syst Rev 2016;5:CD012189.

15 The National Confidential Inquiry into Suicide and Safety in Mental Health (NCISH). Suicide by middle-aged men. Manchester: University of Manchester, 2021.

16 Bakolis I, Stewart R, Baldwin D, et al. Changes in daily mental health service use and mortality at the commencement and lifting of COVID-19 'lockdown' policy in $10 \mathrm{UK}$ sites: a regression discontinuity in time design. BMJ Open 2021;11:e049721.

17 Morgan C, Webb RT, Carr MJ, et al. Incidence, clinical management, and mortality risk following self harm among children and adolescents: cohort study in primary care. BMJ 2017;359:j4351.

18 Hunt IM, Richards N, Bhui K. Suicide rates by ethnic group among patients in recent contact with mental health services: an observational study in England and Wales, 2007-2018. Lancet Psychiatry 2021.

19 Carr MJ, Ashcroft DM, Kontopantelis E, et al. The epidemiology of self-harm in a UK-wide primary care patient cohort, 2001-2013. BMC Psychiatry 2016;16:53.

20 Taylor AK, Steeg S, Quinlivan L, et al. Accuracy of individual and combined risk-scale items in the prediction of repetition of self-harm: multicentre prospective cohort study. BJPsych Open 2021;7:e2:1-10.

21 The University of Manchester. NIHR greater Manchester patient safety translational research centre. Available: http:// www.patientsafety.manchester.ac.uk/research/themes/safetymarginalised-groups/

22 Suicide prevention national transformation programme. Available: https://www.rcpsych.ac.uk/improving-care/nccmh/ national-suicide-prevention-programme

23 Littlewood DL, Quinlivan L, Graney J, et al. Learning from clinicians' views of good quality practice in mental healthcare services in the context of suicide prevention: a qualitative study. BMC Psychiatry 2019;19:346.

24 Quinlivan L, Littlewood DL, Webb RT, et al. Patient safety and suicide prevention in mental health services: time for a new paradigm? J Ment Health 2020;29:1-5. 